\title{
QR Codes Advantages and Dangers
}

\author{
Krassie Petrova ${ }^{1}$, Adriana Romanello ${ }^{2}$, B. Dawn Medlin ${ }^{3}$ and Sandra A. Vannoy ${ }^{4}$ \\ ${ }^{1}$ Department of Computer Information Systems, School of Engineering, Computer and Mathematical Sciences, \\ Auckland University of Technology, Auckland, New Zealand \\ ${ }^{2}$ Department of Management, Universidad Rey Juan Carlos, Madrid, Spain \\ ${ }^{3}$ Department of Information Systems, Appalachian State University, Boone, U.S.A. \\ ${ }^{4}$ Department of Computer Information Systems, Appalachian State University, Boone, U.S.A.
}

Keywords: $\quad$ QR Codes, QR Characteristics, QR Advantages, QR Dangers.

\begin{abstract}
Quick Response (QR) codes are two dimensional (matrix) codes that have been developed in ways that allow companies and individuals to sell or market their products, skills, and events quickly and easily. The code can be used to represent data such as a web address or map location that can be scanned quickly by a mobile device such as a smart phone. Tracking inventories and marketers were some of the first uses and users of QR codes because of the ease of deployment and low development cost. The use has also grown quickly amongst individual users who want to transfer information such as sending an invitation, providing details about an event, or even announcing a baby's birth. While there are many advantages in the use of QR codes, there is a negative side that has resulted in end users discontinuing their use. Our paper discusses both the usefulness of QR codes as well as the inconveniences and dangers that they may pose.
\end{abstract}

\section{INTRODUCTION}

The symbol known as a Quick Response (QR) code is a two dimensional (matrix) that was introduced in 1994, initially as a means to track parts used in automobile manufacturing in Denso Wave - one of Japan's Toyota group of companies (Brindha and Gopikaarani, 2014). The patent was made available for public use and an international standard was approved by the International Standards organization in 2000 and is regularly being updated (ISO, 2015).

Similarly to the original use of QR codes in the automobile industry, QR codes can be used in a number of areas as a means to support interaction in controlled situations such as in education. Examples include marking assessment (Bhaturkar and Bagde, 2014), providing formative feedback in class (Susono and Shimomura, 2006), and protecting the integrity of online examinations (Soman et al., 2013). In the scenarios above the $\mathrm{QR}$ code is created by the "controlling" central party (e.g., the teacher) and distributed too many client users (students), either the same to all, or personalized to a degree.

There are a number of commercial and free tools that can be used by the central party to generate a QR code (Wainwright, 2015). On the side of the client, users need to have a device (mobile phone) that once the code is made available, the device can read. Second, there must be a means to decode it and obtain the content - either by software that is pre-installed on the client device (e. g., a suitable mobile application), and/or by accessing a remote server to obtain the content or the parts of it that may need user authentication.

Compared to the linear (one-dimensional) bar code the QR code has two major functional advantages (Rouillard, 2008). First, due to the high data-density of the encoding (approximately 100 times more than a bar code) a QR code can contain significantly more information than a bar code while occupying a comparable space slot (up to 7000 alphanumeric characters), and secondly, it is able to support encoding of characters such as the ones used in logographic and phonemic writing systems (e. g., Kanji and Kana).

This position paper acknowledges the advantages of QR codes; however, the current environment of hacking and identify theft has highlighted this symbol (QR) as a means to capture information and to create fraudulent activities. Additionally, dangerous and malicious QR codes have been created to direct end users to sites that contain malicious software among 
other types of destructive attacks.

\section{CREATION OF QR CODES}

QR codes can be created easily and cheaply, often at no cost. If an individual does a search on the Internet for a QR code creator, hundreds of links are found that offer free applications.

These tools are simple to use as they allow the end user to easily make selections to create the QR code. The creator can select where the QR takes the reader. It can be to a URL, phone number, SMS, text, maps or other locations.

Attributes that may be set within the code include the size of the $Q R$ code, such as $S$ : One square inch (a perfect size for your business card), and as well as its color.

As an example, the QR code below was created using QRStuff.com, a free tool that links you to the website of Appalachian State University.

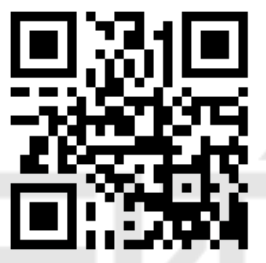

Figure 1: QR code to Appalachian State University.

Creation of QR codes are continually being developed with new inclusions of symbols and different shapes, but issues have arisen such as errors around the decoding process. But there have been successes.

A novel image blending method of improving the QR code visual significance for marketing purposes is proposed by Baharav and Kakarala (2013); it improves QR code aesthetics and visual significance by embedding images such as brand logos in full colour, without a negatively impacting the error correction. Furthermore, (Lin et al., 2013a) proposed a systematic QR code beautification framework that allows an individual user to personalize the $\mathrm{QR}$ code they create (for example a QR code containing contact details meant to be to be printed on a business card) by selecting visually meaningful patterns.

\section{QR CODE ADOPTION}

Despite the promising opportunities QR code use by individuals is not widely spread worldwide except for Japan and Korea (Sasaki et al., 2007, Rouillard, 2008) but was well established in Japan and in Korea (Ryu and Murdock, 2013, Sago, 2011). More specifically Sago (2011) found that the participants in their research (college students) did not fully understand how QR codes worked and were not sure whether their mobile phones could read them; therefore, they were not interested in reading a $\mathrm{QR}$ code when seeing one. However participants who showed interest in QR codes were highly likely to use them in the future. Similar results were obtained in more recent work (Ozkaya et al., 2015, Lo, 2014) which also found that even individuals who considered themselves innovative were not very likely to use QR codes.

The findings in these studies and also in Okazaki et al. (2012) and Jung et al. (2012) indicated that the perceived usefulness and ease of use of QR code as well as perceived attractiveness may influence positively user attitude towards QR codes; a relationship between the type of product being marketed and expected QR use was identified by Narang et al. (2012).

Addressing in part the noticeable lack of increase in customer adoption of QR use, current advances in QR development has focused on improving their appeal. A novel image blending method of improving the QR code visual significance for marketing purposes is proposed by Baharav and Kakarala (2013); it improves QR code aesthetics and visual significance by embedding images such as brand logos in full colour, without a negative impact the error correction. Furthermore (Lin et al., 2013a) propose a systematic QR code beautification framework that allows an individual user to personalize the QR code they create (for example a QR code containing contact details meant to be to be printed on a business card) by selecting visually meaningful patterns.

Unfortunately, all of these advances to make QR codes more appealing to end users may make them more appealing to hackers and those that want to use them in harmful and menacing ways.

\section{ADVANTAGES OF QR CODES}

There are a number of other use contexts that involve creating as well using QR codes by individuals as a tool to transfer information as described by Narayanan (2012). Examples include encoding personal details in a QR code for others to scan and decode on their devices or scanning someone's QR code to load their details on the reader's phone (i.e., using the QR code as a machine readable personal card), sending and receiving invitations (i.e., 
encoding detail about an event including location in a QR code which can be posted on Web pages, or printed in other media, to be scanned by people who want to obtain the invitation).

As an overview of advantages, they include the following:

- Can decide the action you want the customer to take.

- Follow ISO standards.

- Completely measureable.

- Instant information available to consumers.

- Reduces reprints of advertising materials, and

- Is an established marketing tool.

These advantages and ease of use of QR codes have found new adopters. New adopters, and all users may not be aware that cyber criminals use these same applications to introduce "malicious QR codes."

\section{DANGERS OF QR CODES}

The ease with which one can create and distribute QR codes has not only attracted businesses, but the criminal element as well. QR Codes, like many other mobile applications, have been developed with little forethought to security. While most of us will think twice about opening a questionable email or visiting an uncertain website, we often have no qualms about scanning a QR code. Most people are unaware that scanning an unknown QR code offers serious security concerns. While the QR code itself isn't dangerous, there is no opportunity to evaluate the site it will lead you to such as the case with an email or website. If the barcode application displays the URL, an observant user may notice a suspicious-looking URL. However, URL shorteners can make it more difficult for users to evaluate the legitimacy of a URL (Vidas et al., 2012). Typically, the end user reads the code without evaluating risks, and then suffers the consequences if there are security problems.

It is quite easy for a sticker to be printed containing a malicious QR code and then attached over the legitimate code, a type of attack that is known as attagging. QR codes are the perfect vehicle for malicious attacks, facilitating phishing (QRishing) attacks and redirecting users to malicious websites that host viruses, worms, and Trojans (Jain and Shanbhag, 2012). Malicious embedded URLs can lead to malware being installed on mobile devices and result in the loss of sensitive personal data and even damage to software and hardware (Narayanan, 2012).
When a user takes a photo of a QR code, the link it stores is first displayed on the device's screen; however, cybercriminals also use URL shortening services (such as bit.ly and others) to disguise the ultimate address stored in the QR code which may lead to a page with malware that steals the user's credentials or to a phishing site (Malenkovich, 2015).

QR codes are seen in magazines, on billboards, and on storefronts. They seem to be anywhere and everywhere. Because of the unique ability of $\mathrm{QR}$ codes to bridge the gap between virtual reality and actual reality, many consumers forget that QR codes pose the same dangers as emails and websites that can have the ability to capture personal information.

The general design of $\mathrm{QR}$ codes makes it impossible to distinguish one from another with the human eye, meaning that anyone can replace legitimate codes with an illegitimate one using a sheet of QR coded stickers. In Russia, cybercriminals used imposter QR codes to siphon cash and personal information from hundreds of smartphone owners in 2011 and were refining their methods to dupe even more users.

\section{RECOMMENDATIONS}

Several recommendations can be made with regard to security and QR code scanning. For example, companies such as Norton are developing products to help combat security issues around QR code. QR code reader Norton Snap verifies the safety of websites before they are allowed to load on your mobile device. When the QR code is read, the website's safety rating is checked and the application will block any suspicious sites. It also allows the user to view the URL. QR Pal, another secure QR code reader, utilizes SafeScan, a built-in fraud prevention technology, to alert the end user when it detects fraudulent activity associated with a scan. It is recommended that only $\mathrm{QR}$ code readers that allow evaluation of the URL before directing the user to a site should be used.

As QR codes often lead to virus-infected sites, the mobile user should always use an antivirus app. But this is also an issue as smart phone users do not generally adopt security measures such as the use of anti-virus apps. Mobile devices are particularly susceptible to viruses, and should be actively protected. The most effective security measure is to carefully evaluate a QR code before scanning. Only scan codes from trusted sources. For example, while scanning a QR code in a magazine should be relatively secure, scanning a code on a handmade 
flyer would not be wise. Additionally, before scanning a code from a public venue, it would be wise to simply feel the code to ensure that it is not a sticker, which could indicate a security risk ("Scanning QR Codes: Be safe").

\section{CONCLUSIONS}

The general design of a QR code makes it easy and cheap to create, as well as impossible to identify as a "good" QR code versus a "harmful" one. But there are steps that companies and individuals must assume before simply scanning a random $\mathrm{QR}$ code and trusting that it will not introduce malicious code or do harm rather than good. Simply put, end users should never trust any QR code and be suspicious and alert to where any QR code may take them.

\section{REFERENCES}

Baharav, Z. \& Kakarala, A, R. 2013. Visually significant QR codes: Image blending and statistical analysis. Multimedia and Expo (ICME), 2013 IEEE International Conference on, 2013. IEEE, 1-6.

Bhaturkar, K. P. \& Bagde, K. G. 2014. QR code based digitized marksheet. International Journal of Management, IT and Engineering, 4, 57.

Brindha, G. \& Gopikaarani, N, 2014. Secure banking using QR code.

ISO .2015. Available: http://www.iso.org/iso/catalogue detail?csnumber $=43655$ [Accessed 1 March 2016].

Jain, A. K., \& Shanbhag, D,2012. Addressing security and privacy risks in mobile applications. IT Professional, (5), 28-33.

Jung, J.-H., Somerstein, R. \& Kwon, E. S., 2012. Should i scan or should i go?: Young consumers'motivations for scanning qr code advertising. International Journal of Mobile Marketing, 7.

Lin, Y.-H., Chang, Y.-P. \& Wu, J.-L., 2013a. Appearancebased QR code beautifier. Multimedia, IEEE Transactions on, 15, 2198-2207.

Lo, H., 2014. Quick response codes around us: Personality traits, attitudes toward innovation, and acceptance. Journal of Electronic Commerce Research, 15, 35-39.

Malenkovich, 2015. Retrieved from https://blog. kaspersky.com/qr-codes-convenient-dangerous/1115/ on April 8, 2015.

Moore, R., Lopes, J., 1999. Paper templates. In TEMPLATE'06, 1st International Conference on Template Production. SCITEPRESS.

Narang, S., Jain, V. \& Roy, S. 2012. Effect of QR codes on consumer attitudes. International Journal of Mobile Marketing, 7, 52-64.
Narayanan, S. (2012), QR Codes and Security Solutions. International Journal of Computer Science and Telecommunications, 3(7), 69-72.

Okazaki, S., Li, H. \& Hirose, M., 2012. Benchmarking the use of QR code in mobile promotion. Journal of Advertising Research, 52, 102-117.

Ozkaya, E., Ozkaya, H. E., Roxas, J., Bryant, F. \& Whitson, D., 2015. Factors affecting consumer usage of QR codes. Journal of Direct, Data and Digital Marketing Practice, 16, 209-224.

Rouillard, J., 2008. Contextual QR Codes. Proceedings of the Third International Multi-Conference on Computing in the Global Information Technology (ICCGI 2008). IEEE.

Ryu, J. S. \& Murdock, K. 2013., Consumer acceptance of mobile marketing communications using the QR code. Journal of Direct, Data and Digital Marketing Practice, 15, 111-124.

Sago, B., 2011. The usage level and Effectiveness of Quick Response (QR) codes for integrated marketing communication purposes among college students. International Journal of Integrated Marketing Communications, 3, 7-17.

Sasaki, J., Shimomukai, H., Yoneda, T. \& Funyu, Y., 2007. Development of Designed QR Code. Frontiers in Artificial Intelligence and Applications, 154, 290.

"Scanning QR Codes: Be Safe." Retrieved from http://beqrioustracker.com/scanning-qr-codes-be-safe/ on April 8, 2016.

Soman, N., Shelke, U. \& Patel, S., 2013. Automated Examination Using QR Code. International Journal of Engineering and Advanced Technology, 2, 622-627.

Susono, H. \& Shimomura, T., 2006. Using mobile phones and QR codes for formative class assessment. Current developments in technology-assisted education, 2, 1006-1010.

Vidas, T., Owusu, E., Wang, S., Zeng, C., Cranor, L, 2012 QRishing: The Susceptibility of Smartphone Users to QR Code Phishing Attacks. CyLab: Carnegie Mellon University, 12 pgs.

Wainwright, C., 2015. How to make a QR code in 4 quick steps [Online]. Available: http://blog.hubspot.com/ blog/tabid/6307/bid/29449/How-to-Create-a-QRCode-in-4-Quick-Steps.aspx [Accessed 1 March 2016].

Zhou, Y. \& Jiang, X., 2012. "Dissecting Android Malware: Characterization and Evolution," 2012 IEEE Symposium on Security and Privacy, San Francisco, CA, pp. 95-109. 